St ochast i c and deter mi ni stic absor pt i on i n si ngl e- phot on i nt erfer ence exper i ment s

\begin{tabular}{|l|l|}
\hline 著者 & Awaya Kayoko, Tomi t a Nakot o \\
\hline $\begin{array}{l}\text { j our nal or } \\
\text { publ i cat i on t i t I e }\end{array}$ & Physi cal Revi ew A \\
\hline vol une & 56 \\
\hline nunber & 5 \\
\hline page range & $4106-4110$ \\
\hline year & $1997-11$ \\
\hline 出版者 & Aner i can Physi cal Soci et y \\
\hline 権利 & (c) 1997 The Aner i can Physi cal Soci et y \\
\hline URL & ht t p: //hdl . handl e. net /10297/587 \\
\hline
\end{tabular}




\title{
Stochastic and deterministic absorption in single-photon interference experiments
}

\author{
Kayoko Awaya \\ Faculty of Environmental and Information Sciences, Yokkaichi University, Yokkaichi Mie 512, Japan \\ Makoto Tomita \\ Department of Physics, Faculty of Science, Shizuoka University, Ohya Shizuoka 422, Japan
}

(Received 21 January 1997; revised manuscript received 10 April 1997)

\begin{abstract}
We performed single-photon interference experiments with neutral density filters and slow beam choppers to confirm the difference between stochastic and deterministic absorption and obtained results that agreed with the case of neutron-interference experiments and quantum theory. [S1050-2947(97)05810-1]

PACS number(s): 42.50.Ct, 42.50.Ar
\end{abstract}

\section{INTRODUCTION}

Quantum theory is composed of two fundamental procedures. Namely, between measurements an object is described by the Schrödinger time development, while in a measurement we have to apply the statistical formula to it. Whether these two procedures are consistently unified or not has been a subject of debate since von Neumann's theory of measurement [1].

Recently, Rauch and Summhammer performed MachZehnder-type interference experiments with a thermal neutron beam to expose two types of interference patterns [2]. They correspond to the two types of absorbers in one path of the neutron interferometer and they are called stochastic and deterministic absorbers, respectively. Through the stochastic absorber where partially absorbing foils are used, the wave function of a neutron changes by the Schrödinger time development. But through the deterministic absorber where slow beam choppers are used, the statistical formula has to be used. Their experiments were made under the condition that the probability of finding two or more neutrons inside the interferometer is low enough that they could use a singleparticle wave function, and their result agreed with quantum theory. That is, the normalized interference amplitudes (NIAs) are the square-root dependence $\sqrt{a}$ for the stochastic case and the linear dependence $a$ for the deterministic case, on the same transmission probability $a$ of each absorber. Here the slow beam chopper means that the open-closed period of the chopper is much longer than the coherence time of the neutron's wave packet. So there would occur a question what happens when the chopper becomes very fast in rotation. In fact, Summhammer et al. [2] inferred that a onedimensional macroscopic lattice could simulate the fast case of a chopper. However, it is not self-evident whether the fast beam chopper can be simulated by a one-dimensional macroscopic lattice or not.

To clarify this problem we have been making the same kind of experiment with the laser beam under the condition where the average number of photons inside the interferometer is smaller than one. There have been various kinds of photon interference experiments done that take advantage of lasers [3-8]. This paper gives a report on stochastic and deterministic absorption in single-photon interference experiments. Our experimental results agreed with the case of the neutron interferometer and quantum theory. Although the results can be described with a classical electric field, if we analyze the experiment in terms of photons, the interpretation is an interesting one and we have demonstrated via a simple experiment the difference between the slow chopper with mean transmission $a$ and a neutral density (ND) filter with similar mean transmission.

\section{THEORY}

If one can approximately describe a system as a singlephoton state inside the interferometer, then the probability of detecting the single photon at $(\mathbf{r}, t)$ on the detector is proportional to [9]

$$
\left\langle\Psi\left|\hat{E}^{(-)}(\mathbf{r}, t) \hat{E}^{(+)}(\mathbf{r}, t)\right| \Psi\right\rangle
$$

because of the electric-dipole interaction on the photoelectric effect at the detector. Here $\hat{E}^{(+)}(\mathbf{r}, t)$ and $\hat{E}^{(-)}(\mathbf{r}, t)$ are positive and negative frequency parts of the electric-field operator at $(\mathbf{r}, t)$, and $|\Psi\rangle$ is the initial photon state in the detection process.

In the stochastic case where a ND filter is inserted as the absorber in Fig. 1, the photon state through the two beam splitters and the absorber (the ND filter) with the transmission probability $a$ can be described as

$$
\begin{aligned}
|\Psi\rangle= & \left(\Phi_{A}+\Phi_{B} \sqrt{a}\right)|0\rangle_{C}|1\rangle_{D}+\left(\Phi_{A}^{\prime}+\Phi_{B}^{\prime} \sqrt{a}\right)|1\rangle_{C}|0\rangle_{D} \\
& +\mid \text { o.s. }\rangle
\end{aligned}
$$

by the Schrödinger time development (see the Appendix). Here $\Phi_{A}$ and $\Phi_{B}$ correspond to the wave functions that are brought into the detector (beam $D$ ) through paths $A$ and $B$, respectively, and $\Phi_{A}^{\prime}$ and $\Phi_{B}^{\prime}$ are the wave functions into beam $C$ through paths $A$ and $B$ in the case of no absorber. Photon state $|n\rangle_{X}$ means that the number of photons in beam $X$ (mode $X$ ) is $n$, where we use a fairly monochromatic incident beam. And $\mid$ o.s. $\rangle$ means the sum of all the other states that are removed from beams $C$ and $D$. In the mode expansions of $\hat{E}^{(+)}(\mathbf{r}, t)$ and $\hat{E}^{(-)}(\mathbf{r}, t)$ at the detector, we may consider the annihilation and creation operators of single photons only on beam $D$ (mode $D$ ) for the detection. Thus from Eqs. (1) and (2), the intensity at the detector is 


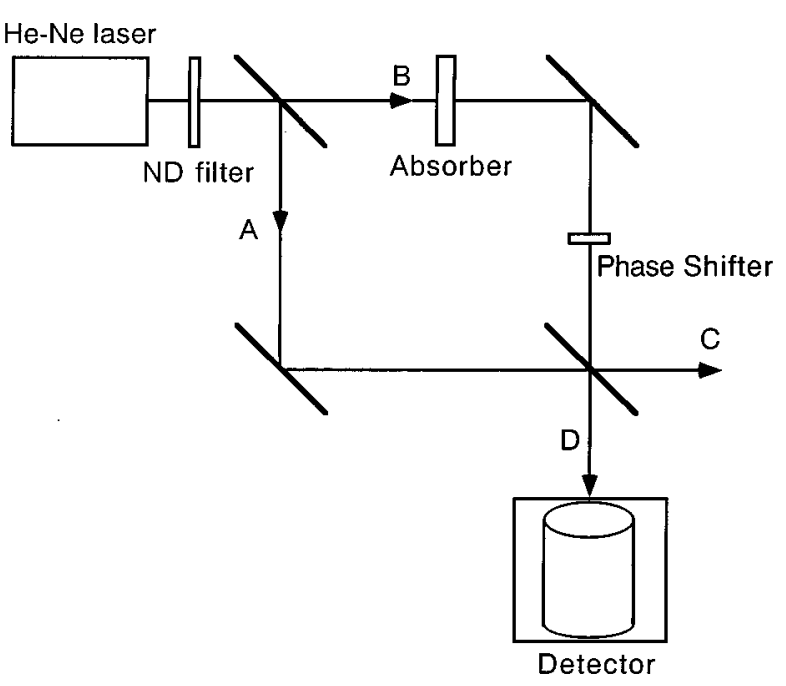

FIG. 1. Setup for the single-photon interference experiments with various kinds of absorbers in path $B$.

$$
I=\left|\Phi_{A}+\Phi_{B} \sqrt{a}\right|^{2}=|\Phi|^{2}\left(1+\rho^{2} a+2 \rho \sqrt{a} \cos \chi\right),
$$

where

$$
\Phi_{A} \equiv \Phi, \quad \Phi_{B}=\rho e^{i \chi} \Phi \quad(\rho, \chi \text { real })
$$

When there is no absorber in the interferometer $(a=1)$, the intensity is

$$
I^{0}=|\Phi|^{2}\left(1+\rho^{2}+2 \rho \cos \chi\right)
$$

Therefore we get the square-root dependence of the NIA in the stochastic case, such as

$$
\begin{aligned}
\operatorname{NIA}(S) & \equiv[I(\max )-I(\min )] /\left[I^{0}(\max )-I^{0}(\min )\right] \\
& =4 \rho \sqrt{a}|\Phi|^{2} / 4 \rho|\Phi|^{2}=\sqrt{a} .
\end{aligned}
$$

In the deterministic case where a slow beam chopper is inserted instead of the ND filter, we have to take the ensemble average of the following two cases. When path $B$ is open, the result is just the same as $I^{0}$ above. But when path $B$ is closed, the single photon is either completely absorbed by the chopper or goes through path $A$. This is the so-called reduction of the wave packet and the probability of going into the detector through path $A$ is $\left|\phi_{A}\right|^{2}$ by the statistical formula of quantum theory. Here the ratio between the opening and closing time of the chopper is $a$ to $1-a$ with the transmission probability $a$ of the chopper. So the intensity at the detector is

$$
\begin{aligned}
I & =a\left|\Phi_{A}+\Phi_{B}\right|^{2}+(1-a)\left|\Phi_{A}\right|^{2} \\
& =|\Phi|^{2}\left(1+\rho^{2} \alpha+2 \rho a \cos \chi\right) .
\end{aligned}
$$

When there is no chopper in the interferometer $(a=1)$, the intensity is

$$
I^{0}=|\Phi|^{2}\left(1+\rho^{2}+2 \rho \cos \chi\right)
$$

Then we get the linear dependence of the NIA in the deterministic case,

$$
\operatorname{NIA}(D)=4 \rho a|\Phi|^{2} / 4 \rho|\Phi|^{2}=a
$$

\section{EXPERIMENT AND RESULTS}

The experimental setup is shown in Fig. 1. We used a Mach-Zehnder interferometer, where the optical path length of each arm is $40 \mathrm{~cm}$. The light source was a $\mathrm{TEM}_{00}$ single mode, average power $1 \mathrm{~mW}$, He-Ne laser (Spectra-Physics $117 \mathrm{~A})$ with a wavelength of $632.8 \mathrm{~nm}$. The coherence length is longer than $5 \mathrm{~km}$. The frequency stability is $\pm 0.5 \mathrm{MHz} / \mathrm{min}$. The incident beam intensity was reduced by a factor of $10^{-7}$ by ND filters so that the mean number of photons inside the interferometer is less than 0.4. If we apply a Poisson distribution with the mean number 0.4 to the photon state of our experimental situation, the probability of finding two or more photons inside the interferometer is 0.062 . So the treatment of the system as a single-photon state is justified. In order to compare the results in stronger beam intensities with ones in single-photon states, we also made experiments using a stronger incident beam increased by a factor of $10^{3}$.

A photomultiplier tube (HAMAMATSU Photonics R374) cooled to $-30{ }^{\circ} \mathrm{C}$ was placed on path $D$. The quantum efficiency of the photomultiplier tube was $7 \%$. The photon pulses from the photomultiplier tube were led to a gated counting board with 50-nsec temporal resolution. The gate time was set to $0.5 \mathrm{sec}$. The mean number of background thermal pulses per $0.5 \mathrm{sec}$ was 15 counts. A 632.8-nm interference filter with a peak transmission probability of $50 \%$ was placed in front of the photomultiplier tube. An optical glass plate with $3 \mathrm{~mm}$ thickness was placed in path $B$ and rotated by using a stepping motor as a phase shifter in all experiments.

The stochastic absorbers consisted of various ND filters so that the transmission probability along path $B$ could be varied between $1 \%$ and $80 \%$. For deterministic absorbers, various choppers with the transmission probability between $8 \%$ and $90 \%$ were used. The choppers consisted of rotating black disks where the necessary number of holes were made. The open-closed frequencies were between 100 and $300 \mathrm{~Hz}$. This corresponds to the case of the slow chopper described above. Figure 2 shows typical interference patterns as a function of rotational angle of the phase shifter. The transmission probability along path $B$ was measured in the same way for both kinds of absorbers: path $A$ was blocked and then the intensities at the detector were recorded with the absorber present in path $B$ and without it.

In Fig. 3 the NIAs are plotted for the different kinds of absorbers together with the theoretical predictions for the stochastic and deterministic cases. The solid circles and triangles are NIAs with absorbers of ND filters and slow beam choppers, respectively, in single-photon experiments. The open circle and triangle are for the same kinds of experiments with the stronger incident beam by a factor of $10^{3}$. The solid and broken lines represent Eqs. (3) and (4), respectively.

\section{CONCLUDING REMARKS}

The results obtained by single-photon experiments agreed with the theoretical predictions in quantum theory within our 


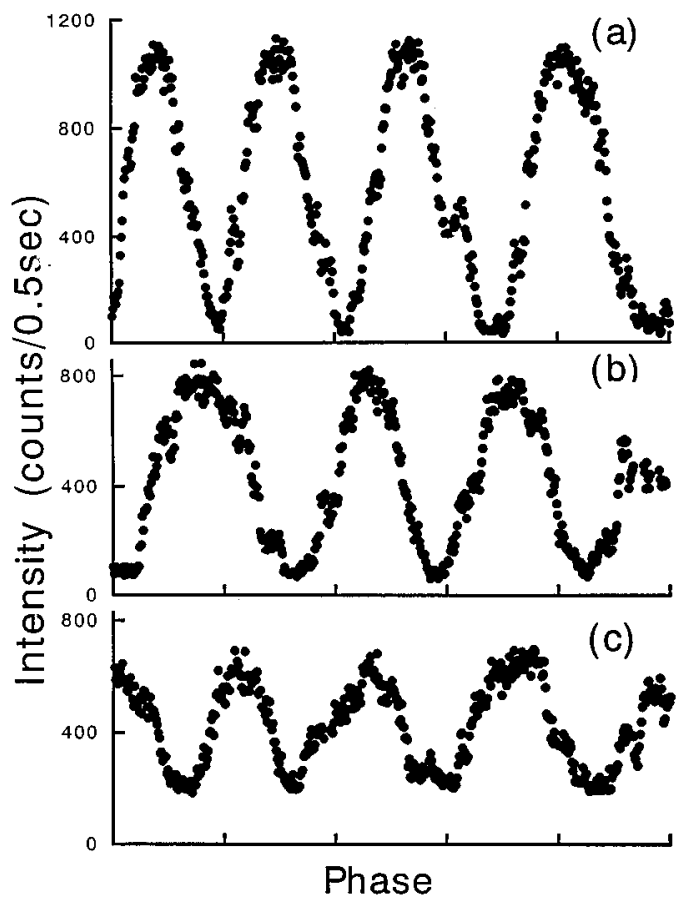

FIG. 2. Examples of the interference patterns as a function of the phase shift: (a) without absorber, (b) with a ND filter of the transmission probability of 0.49 , and (c) with a beam chopper of the transmission probability of 0.50 .

experimental errors. With the stronger incident beam, the results also lie on the corresponding theoretical lines. This is because the interference phenomena in the classical wave are formally the same as the case of the single-photon state [9]. However, the interpretations when path $B$ is closed with the chopper are completely different from each other. Classically, the electric field is continuous and the component along path $B$ is absorbed by the chopper, and only the component along path $A$ is brought into the detector, resulting in

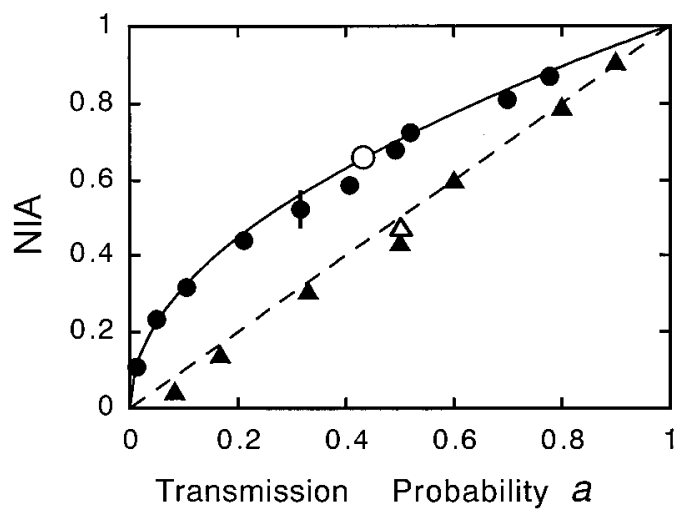

FIG. 3. Normalized interference amplitude (NIA) as a function of transmission probability through path $B$. The solid circles and triangles are with ND filters and choppers, respectively in the single-photon experiments. The open circle and triangle are for the same kinds of experiments with the stronger incident beam by a factor of $10^{3}$. The solid and broken lines represent the theoretical predictions for the stochastic and deterministic absorbers, respectively.

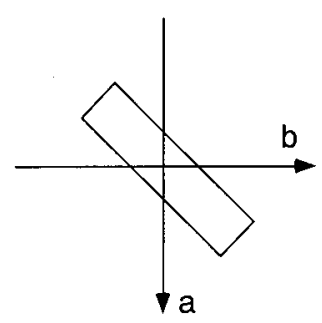

(a)

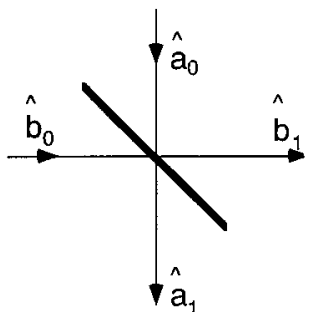

(b)
FIG. 4. (a) Beam splitter with two modes $a$ and $b$. In the Heisenberg representation, the time-dependent mode operators $\hat{a}(t)$ and $\hat{b}(t)$ for a single-photon develop by the interaction with the beam splitter during the time duration for the photon to pass through the beam splitter. (b) Beam splitter with the conventional mode operators.

no interference effect. But a single photon selects either path $A$, resulting in the detection, or path $B$, resulting in no detection, and so a single photon displays particlelike behavior i.e., "which path" information. We get the same result as in classical wave only under the accumulation of many repeated single-photon measurements.

We should note that the reduction of the wave packet by a chopper cannot be described by the Heisenberg representation where the wave packet never change in time. Nor does it seem to be transformed to the Heisenberg representation from the Schrödinger representation, because there is no Hamiltonian and therefore no unitary transformation describing the reduction of the wave packet. We should also note that actually the transmission probability $a$ of a ND filter is the ensemble average of many repeated measurements and the description $\sqrt{a}$ in Eq. (2) can be justified only when the fluctuation of $a$ is sufficiently smaller than $a$ itself as in our experiments; i.e., $\sqrt{a} / a$ is smaller than 0.03 .

In our experiments, the ratio of the open-closed period of the chopper to the photon coherence time is of the order of $10^{3}$. In the neutron interferometer, the ratio is of the order of $10^{10}$ and it looks difficult to get a faster chopper because of mechanical vibrations [2]. But it seems rather feasible for the photon interferometer to get a faster beam chopper. The case of a fast chopper, i.e., the coherence time of a photon comparable to or rather longer than the open-closed period of the chopper, is a subject planned to be studied.

\section{ACKNOWLEDGMENTS}

The authors would like to thank T. Nishioka and T. Ishigame for their help during the measurements.

\section{APPENDIX: QUANTUM THEORY OF THE BEAM SPLITTER}

We consider a single-photon state with monochromatic angular frequency $\omega$ and two modes $a$ and $b$ entering a beam splitter shown in Fig. 4(a). We treat this process by suppos- 
ing that the interaction between a single photon and the beam splitter is

$$
\hat{H}_{I}=-(\alpha h / 2 \pi)\left(\hat{a}^{\dagger} \hat{b}+\hat{b}^{\dagger} \hat{a}\right)
$$

where $\alpha$ is a real coupling constant and $h$ is Planck's constant, and the total Hamiltonian for the photon in the beam splitter is

$$
\hat{H}=\hat{H}_{0}+\hat{H}_{I},
$$

with

$$
\hat{H}_{0}=(\omega h / 2 \pi)\left(\hat{a}^{\dagger} \hat{a}+\hat{b}^{\dagger} \hat{b}\right) .
$$

The creation and annihilation operators $\hat{a}^{\dagger}, \hat{b}^{\dagger}$ and $\hat{a}, \hat{b}$ for the modes $a$ and $b$ satisfy the following equations:

$$
\begin{gathered}
{\left[\hat{a}, \hat{a}^{\dagger}\right]=\left[\hat{b}, \hat{b}^{\dagger}\right]=1,} \\
{[\hat{a}, \hat{b}]=\left[\hat{a}, \hat{b}^{\dagger}\right]=0}
\end{gathered}
$$

for any given time. Then in the Heisenberg representation, we have

$$
\begin{aligned}
& d \hat{a} / d t=(i 2 \pi / h)[\hat{H}, \hat{a}]=i \alpha \hat{b}-i \omega \hat{a}, \\
& d \hat{b} / d t=(i 2 \pi / h)[\hat{H}, \hat{b}]=i \alpha \hat{a}-i \omega \hat{b} .
\end{aligned}
$$

So

$$
\begin{gathered}
\hat{a}(t)-\hat{b}(t)=\left\{\hat{a}\left(t_{0}\right)-\hat{b}\left(t_{0}\right)\right\} e^{-i(\alpha+\omega)\left(t-t_{0}\right)}, \\
\hat{a}(t)+\hat{b}(t)=\left\{\hat{a}\left(t_{0}\right)+\hat{b}\left(t_{0}\right)\right\} e^{i(\alpha-\omega)\left(t-t_{0}\right)},
\end{gathered}
$$

resulting in

$$
\begin{aligned}
& \hat{a}(t)=e^{-i \omega\left(t-t_{0}\right)}\left\{\hat{a}\left(t_{0}\right) \cos \alpha\left(t-t_{0}\right)+i \hat{b}\left(t_{0}\right) \sin \alpha\left(t-t_{0}\right)\right\}, \\
& \hat{b}(t)=e^{-i \omega\left(t-t_{0}\right)}\left\{i \hat{a}\left(t_{0}\right) \sin \alpha\left(t-t_{0}\right)+\hat{b}\left(t_{0}\right) \cos \alpha\left(t-t_{0}\right)\right\} .
\end{aligned}
$$

Thus if we suppose that the interaction $\hat{H}_{I}$ is switched on at $t=t_{0}$ and off at $t=t_{0}+T$, where $T$ is the time duration for the photon to pass through the beam splitter, then, for $t<t_{0}$,

$$
\begin{gathered}
\hat{a}\left(t<t_{0}\right)=e^{-i \omega t} \hat{a}_{0}, \\
\hat{b}\left(t<t_{0}\right)=e^{-i \omega t} \hat{b}_{0}
\end{gathered}
$$

and for $t>t_{0}+T$,

$$
\begin{aligned}
& \hat{a}\left(t>t_{0}+T\right)=e^{-i \omega t}\left\{\hat{a}_{0} \cos \alpha T+i \hat{b}_{0} \sin \alpha T\right\} \equiv e^{-i \omega t} \hat{a}_{1}, \\
& \hat{b}\left(t>t_{0}+T\right)=e^{-i \omega t}\left\{i \hat{a}_{0} \sin \alpha T+\hat{b}_{0} \cos \alpha T\right\} \equiv e^{-i \omega t} \hat{b}_{1} .
\end{aligned}
$$

This results in the conventional relations between the incoming and outgoing mode operators, $\hat{a}_{0}, \hat{b}_{0}$ and $\hat{a}_{1}, \hat{b}_{1}$ shown in Fig. 4(b), i.e.,

$$
\hat{a}_{1}=\hat{a}_{0} \cos \alpha T+i \hat{b}_{0} \sin \alpha T,
$$

$$
\hat{b}_{1}=i \hat{a}_{0} \sin \alpha T+\hat{b}_{0} \cos \alpha T
$$

where $(\cos \alpha T)^{2}$ and $(\sin \alpha T)^{2}$ correspond to the transmission and reflection probabilities of the beam splitter, respectively.

In the Schrödinger representation, the photon state is described as

$$
\begin{aligned}
|\Psi(t)\rangle & =e^{-i \hat{H}\left(t-t^{\prime}\right) 2 \pi / h}\left|\Psi\left(t^{\prime}\right)\right\rangle \\
& =e^{-i \hat{H}_{I}\left(t-t^{\prime}\right) 2 \pi / h} e^{-i \hat{H}_{0}\left(t-t^{\prime}\right) 2 \pi / h}\left|\Psi\left(t^{\prime}\right)\right\rangle
\end{aligned}
$$

because of $\left[\hat{H}_{I}, \hat{H}_{0}\right]=0$. When the initial state is a singlephoton state in mode $a$, it can be described as

$$
\left|\Psi\left(t<t_{0}\right)\right\rangle=e^{-i \omega t}|1\rangle_{a}|0\rangle_{b}
$$

because $\hat{H}_{I}=0$ for $t<t_{0}$. Then

$$
e^{-i \hat{H}_{0}\left(t-t_{0}\right) 2 \pi / h}\left|\Psi\left(t_{0}\right)\right\rangle=e^{-i \omega t}|1\rangle_{a}|0\rangle_{b}
$$

and

$$
\begin{aligned}
\left|\Psi\left(t_{0}+T\right)\right\rangle= & e^{-i \omega\left(t_{0}+T\right)} e^{-i \hat{H}_{I} T 2 \pi / h}|1\rangle_{a}|0\rangle_{b}=e^{-i \omega\left(t_{0}+T\right)} \\
& \times\left\{|1\rangle_{a}|0\rangle_{b} \cos \alpha T+i|0\rangle_{a}|1\rangle_{b} \sin \alpha T\right\}
\end{aligned}
$$

Thus

$$
\begin{aligned}
\mid \Psi(t & \left.\left.>t_{0}+T\right)\right\rangle=e^{-i \hat{H}_{0}\left(t-t_{0}-T\right) 2 \pi / h}\left|\Psi\left(t_{0}+T\right)\right\rangle \\
& =e^{-i \omega t}\left\{|1\rangle_{a}|0\rangle_{b} \cos \alpha T+i|0\rangle_{a}|1\rangle_{b} \sin \alpha T\right\} .
\end{aligned}
$$

In a Mach-Zehnder interferometer shown in Fig. 1, we have two beam splitters, and in general path $A$ and path $B$ are not optically identical. So at the second beam splitter, we can write the initial state of the photon as

$$
\beta_{A}|1\rangle_{A}|0\rangle_{B}+\beta_{B}|0\rangle_{A}|1\rangle_{B}
$$

(from now on we neglect the common overall timedependent phase factor $e^{-i \omega t}$ ). Repeating the same discussion at the second beam splitter, for the initial states $|1\rangle_{A}|0\rangle_{B}$ and $|0\rangle_{A}|1\rangle_{B}$, we get

$$
\begin{gathered}
|1\rangle_{A}|0\rangle_{B} \rightarrow|0\rangle_{C}|1\rangle_{D} \cos \alpha^{\prime} T^{\prime}+i|1\rangle_{C}|0\rangle_{D} \sin \alpha^{\prime} T^{\prime}, \\
|0\rangle_{A}|1\rangle_{B} \rightarrow|1\rangle_{C}|0\rangle_{D} \cos \alpha^{\prime} T^{\prime}+i|0\rangle_{C}|1\rangle_{D} \sin \alpha^{\prime} T^{\prime}
\end{gathered}
$$

by the Schrödinger time development where $\left(\cos \alpha^{\prime} T^{\prime}\right)^{2}$ and $\left(\sin \alpha^{\prime} T^{\prime}\right)^{2}$ correspond to the transmission and reflection probabilities of the second beam splitter, respectively. Thus the final state of the photon through the interferometer is

$$
|\Psi\rangle=\left(\Phi_{A}+\Phi_{B}\right)|0\rangle_{C}|1\rangle_{D}+\left(\Phi_{A}^{\prime}+\Phi_{B}^{\prime}\right)|1\rangle_{C}|0\rangle_{D}
$$

where

$$
\begin{array}{ll}
\Phi_{A}=\beta_{A} \cos \alpha^{\prime} T^{\prime}, & \Phi_{B}=i \beta_{B} \sin \alpha^{\prime} T^{\prime}, \\
\Phi_{A}^{\prime}=i \beta_{A} \sin \alpha^{\prime} T^{\prime}, & \Phi_{B}^{\prime}=\beta_{B} \cos \alpha^{\prime} T^{\prime} .
\end{array}
$$

Here $\beta_{A}$ and $\beta_{B}$, and so $\Phi_{A}$ and $\Phi_{B}$ include the effect through paths $A$ and $B$ with a phase shifter. 
[1] J. von Neumann, Mathematical Foundations of Quantum Mechanics, translated by R. T. Beyer (Springer-Verlag, Berlin, 1932).

[2] H. Rauch and J. Summhammer, Phys. Lett. 104A, 44 (1984); J. Summhammer, H. Rauch, and D. Tuppinger, Phys. Rev. A 36, 4447 (1987).

[3] R. L. Pfleegor and L. Mandel, Phys. Rev. 159, 1084 (1967).

[4] W. Schleich and H. Walther, in Proceedings of the Second International Symposium on Foundations of Quantum Mechanics, edited by M. Namiki et al. (The Physical Society of Japan, Tokyo, 1987), p. 25.
[5] R. Ghosh and L. Mandel, Phys. Rev. Lett. 59, 1903 (1987).

[6] P. G. Kwiat, W. A. Vareka, C. K. Hong, H. Nathel, and R. Y. Chiao, Phys. Rev. A 41, 2910 (1990).

[7] Z. Y. Ou, X. Y. Zou, L. J. Wang, and L. Mandel, Phys. Rev. A 42, 2957 (1990).

[8] L. J. Wang, X. Y. Zou, and L. Mandel, Phys. Rev. A 44, 4614 (1991).

[9] See, for example, R. Loudon, The Quantum Theory of Light, 2nd ed. (Clarendon, Oxford, 1983). 